\title{
Do Airstream Mechanisms Influence Tongue Movement Paths?
}

\author{
Philip Hoole ${ }^{\mathrm{a}}$, Kevin Munhall ${ }^{\mathrm{c}}$, Christine Mooshammer ${ }^{\mathrm{b}}$ \\ a Institut für Phonetik und sprachliche Kommunikation, Ludwig-Maximilians-Universität \\ München, und \\ b Forschungsschwerpunkt allgemeine Sprachwissenschaft, Berlin, Deutschland; \\ c Department of Psychology, Queen's University, Kingston, Ontario, Canada
}

\section{Abstract}

Velar consonants often show an elliptical pattern of tongue movement in symmetrical vowel contexts, but the forces responsible for this remain unclear. We here consider the role of overpressure (increased intraoral air pressure) behind the constriction by examining how movement patterns are modified when speakers change from an egressive to ingressive airstream. Tongue movement and respiratory data were obtained from 3 speakers. The two airstream conditions were additionally combined with two levels of speech volume. The results showed consistent reductions in forward tongue movement during consonant closure in the ingressive conditions. Thus, overpressure behind the constriction may partly determine preferred movement patterns, but it cannot be the only influence since forward movement during closure is usually reduced but not eliminated in ingressive speech.

\section{Introduction}

The sequence of positions (paths) than an articulator follows during movement can reveal much about its planning and control processes. In this paper we describe data showing the paths of tongue movement during velar and alveolar consonant production. Velar consonants, in particular, are well known for showing forward movement of the tongue during the closure phase, producing an elliptical path in symmetrical VCV sequences [Houde, 1968; Perkell, 1969; Kent and Moll, 1972]. In other words, the path followed by a fleshpoint on the tongue for the movement from $\mathrm{C}$ to $\mathrm{V}_{2}$ does not simply retrace the path followed for movement from $\mathrm{V}_{1}$ to $\mathrm{C}$.

In this respect speech movements appear to contrast with the paths observed for limb movements. For example, the path of the hand in reaching and pointing movements is generally observed to be fairly straight in tasks involving movement from a starting position to a target and then back to the starting position (i.e. in a task analogous to production of a target consonant between two identical vowels).

\section{KARGER \\ Fax +41613061234 \\ E-Mail karger@karger.ch \\ www.karger.com \\ (C) 1998 S. Karger AG, Basel $0031-8388 / 98 / 0553-0131$ $\$ 15.00 / 0$ \\ Accessible online at: \\ http://BioMedNet.com/karger}

Philip Hoole

Institut für Phonetik

Schellingstrasse 3, D-80799 München (Germany)

Tel. +49892180 3149, Fax +49892800362

E-Mail hoole@phonetik.uni-muenchen.de 
In the work presented below we examine one of the possible factors that could contribute to the elliptical patterns in speech, namely overpressure behind the constriction. Evidence from earlier work suggested that overpressure could be a relevant influence. For example, Munhall et al. [1991] found more forward movement in $/ \mathrm{k} / \mathrm{in}$ loud speech, where presumably a higher intraoral air-pressure was present than in normal-intensity speech; Mooshammer et al. [1995] found that forward movement was less for the velar nasal (where overpressure is presumably close to zero) than for velar stops. Recent work by Svirsky et al. [1997] on tongue displacement during bilabial stop closure suggested that downward displacements of up to about $2 \mathrm{~mm}$ could be attributable to air pressure effects (Houde [1968] observed effects of similar magnitude; op. cit., pp. 88-92), but it is not easy to assess the extent to which these findings are applicable to the horizontal tongue movements observed in velar consonants. Firstly, the fact that the tongue is actively involved in forming the closure for velars may be a relevant difference. Secondly, the results of Svirsky et al. 11997] suggested a substantially larger pressure-related displacement for voiced consonants compared to voiceless ones (the greater compliance of the tongue in the voiced case presumably assists supraglottal cavity enlargement for sustaining voicing). Yet the results of Mooshammer et al. [1995] showed that the voiceless velar consonants have greater forward movement of the tongue than the voiced ones.

A further possibility to be entertained is that the elliptical patterns reflect the specific arrangement of muscle force vectors available to generate movement into and out of velar closure. The anatomical geometry of each of the tongue muscles, including the points of origin and insertion and the physiological cross-sectional area, determines how much force can be generated in a given direction. When a number of muscles are active simultaneously as in most tongue movements, the total force generated will be the vector sum of the forces generated along each of the active muscles' lines of action. It may be that tongue raising and tongue lowering have different 'summed' directions of force development because of the geometries of their respective muscles. The observed forward movement, then, is the result of the shift from raising to lowering movement vectors. In addition, when multiple muscles are involved in a given movement, then either the initiation times of horizontal and vertical components may differ [Alfonso and Baer, 1982] or these components may show different phase lags behind a common control signal [Sanguineti et al., 1998]. In the above examples, the greater amount of forward movement in loud versus normal speech, and stops versus nasals, might thus reflect an overall higher level of muscular activation in the cases with more pronounced movement.

In an attempt to disentangle these potential contributions we use here a more drastic manipulation of aerodynamic conditions in the vocal tract, by contrasting ingressive and egressive speech, and by combining these two airflow direction conditions with two levels of speech volume (loud and normal). Thus, if air pressure effects are predominantly involved in shaping the movement paths then 'ingressive + loud intensity' should be more likely to show backward movement than 'ingressive + normal intensity'. On the other hand, if muscular effects of the kind outlined in the previous paragraph are more important, then 'ingressive + loud intensity' should be more likely to show forward movement than 'ingressive + normal intensity'; in other words, one would assume that for loud intensity the muscular system responsible for forming velar closure is activated more vigorously, with its forward movement component outweighing any rearward movement component attributable to air pressure.

132 Phonetica 1998;55:131-146 Hoole/Munhall/Mooshammer 


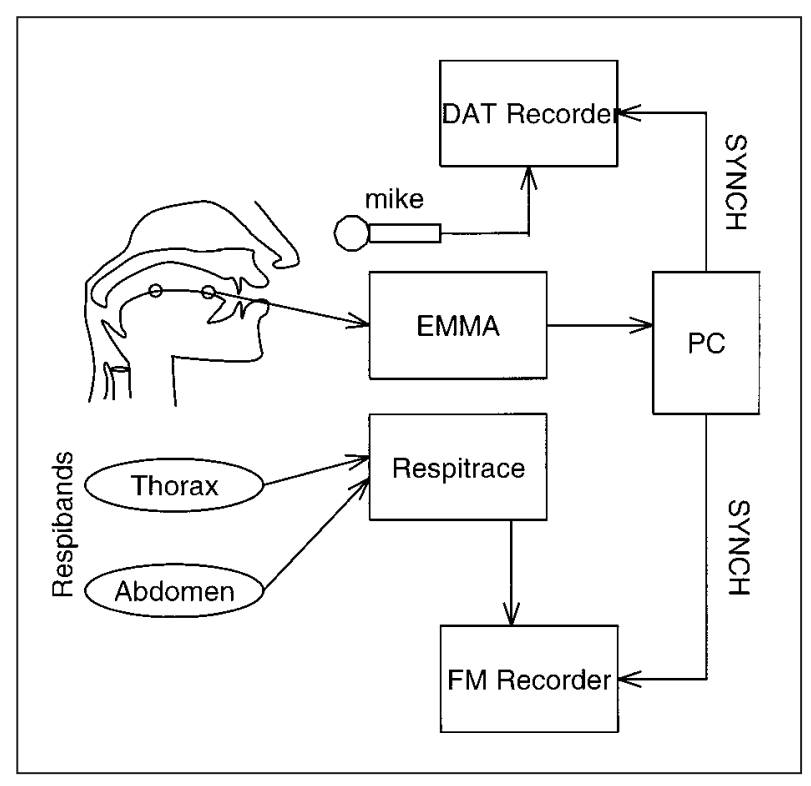

Fig. 1. Experimental setup for simultaneous monitoring of tongue and respiratory movement. EMMA=Electromagnetic midsagittal articulometer; SYNCH= Synchronisation pulse.

Prior to the investigation discussed below we had carried out a pilot investigation of tongue movement in 3 subjects over variation of loudness and airstream direction, but using only a restricted set of utterances (between 16 and $64 \mathrm{VCV}$ sequences). Two subjects showed consistent effects of airstream direction, i.e. less forward movement of the tongue during velar closure when the utterances were spoken on an ingressive airstream. The third subject was not a naive speaker and had been encouraged to practice the sequences before the experiment. He showed negligible airstream influences, suggesting that the effects could be overridden.

The purpose of the more extensive experiment now to be reported was to examine additional naive subjects with a substantially larger number of utterances, and also to simultaneously monitor their respiratory activity in order to aid interpretation of any airstream-related differences in tongue movement patterns.

\section{Procedure}

Material

The speech material consisted of the VCV sequences /ogo/, /igi/, /odo/, /idi/ and /ono/. Each VCV sequence was spoken under 4 airflow conditions: normal intensity and loud intensity, each combined with egressive and ingressive airflow direction.

The 20 speech items $(5 \mathrm{VCV}$ sequences $\times 2$ airflow directions $\times 2$ loudness conditions) were spoken 10 times each in randomized order. At the start of the experiment, the subjects also produced a number of isolated vowels in the different airflow conditions in order to acquaint themselves with the procedure.

Subjects

Three subjects participated: speaker F, English, lecturer in phonetics; speaker M, German, former student of phonetics; speaker S, German, graduate student of phonetics. 


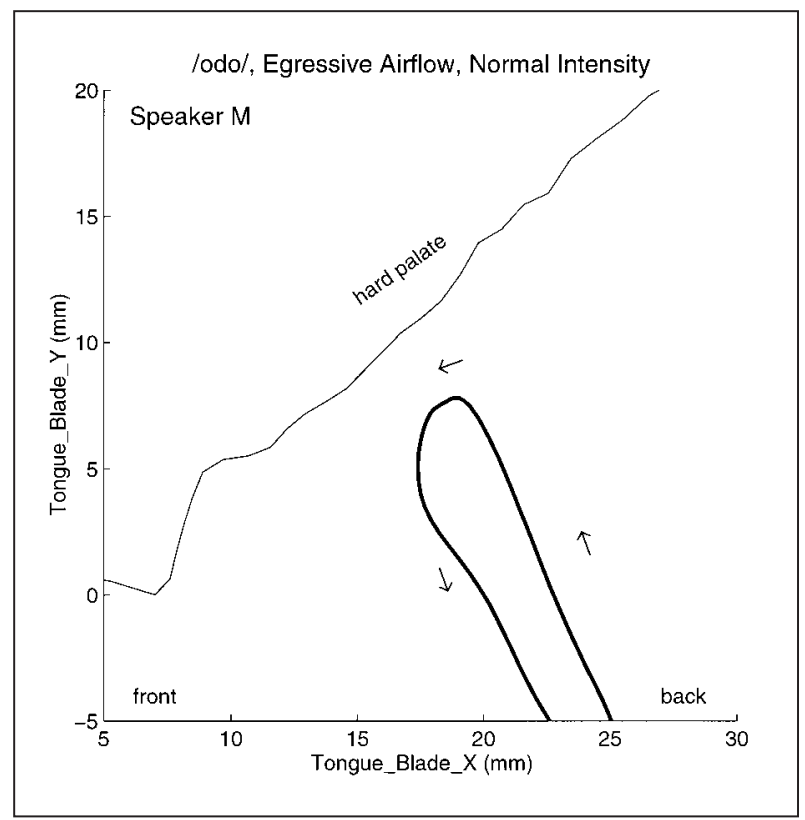

Fig. 2. Example of tongue blade movement relative to the hard palate before rotational normalization.

The subjects were not informed about the purpose of the experiment. They were not told what airflow conditions would be involved until immediately before the start of the experiment. They were not told in advance what specific VCV sequences they would have to produce. Only subjects with phonetic training were used, as it was considered that they should be capable of producing the somewhat unusual material reasonably naturally, and with a minimum of prior explanation and practice.

\section{Recording Procedures}

The experimental setup is shown schematically in figure 1 .

Tongue movement was recorded by means of electromagnetic articulography (EMA; AG100, Carstens Medizinelektronik, Göttingen, Germany). Transducer coils were mounted on the tongue approximately $1 \mathrm{~cm}$ from the tip to monitor alveolar consonant production, and at a point about $1 \mathrm{~cm}$ to the rear of the rear edge of the second molars, with the tongue at rest in the mouth (corresponding to a point about 5-6 $\mathrm{cm}$ from the tip) to monitor velar consonant production. Speaker F had two additional coils, and speakers $\mathrm{M}$ and $\mathrm{S}$ had one additional coil mounted on the tongue between these two, but these will not be considered further here. In addition, reference coils mounted on the upper incisors and on the bridge of the nose were used to compensate for head movement relative to the transmitter coils of the electromagnetic system. The data were rotated so that the horizontal axis was parallel to the occlusal plane of the subject. The EMA system was also used to make tracings of the contour of the hard palate of each subject from dental impressions.

Calibration of the EMA system and assessment of data reliability was carried out using a set of customized procedures developed by the first author (for general discussion of methodological issues in EMA data acquisition, see Perkell et al. [1992] and Hoole [1993, 1996].

In order to provide independent information on the airstream conditions actually produced by the subject, respiratory inductive plethysmography (Respitrace, Ambulatory Monitoring Inc.) was employed to monitor thoracic and abdominal kinematics [Cohn et al., 1978; Bless et al., 1982]. In this way we aimed firstly to check whether the subjects had actually complied with the experimental instructions and secondly to obtain some approximate relative information on average flow rates in the different speech items. 
At the end of the experimental session the subjects performed a number of isovolume maneuvers [Hixon et al., 1973] in order to give some information on how the raw voltage values should be weighted to express equal volumes in the thoracic and abdominal signals. Unfortunately, a faulty lead to one of the Respibands meant that for speaker $\mathrm{F}$ these data, as well as the respiratory data of the last repetition of the speech utterances, could not be evaluated. For speakers $\mathrm{M}$ and $\mathrm{S}$ it was estimated from the isovolume maneuvers that the raw abdominal voltages should be multiplied by 1.61 and 1.27 , respectively, to reflect the same volume contributions as the thoracic signal.

Due to the constraints of the EMA setup, the subjects were sitting during recordings.

\section{Data Analysis}

Following alignment of the audio, EMA and Respitrace signals, data processing proceeded in the following stages.

Preprocessing of Lingual Movement

First of all, the sequences with alveolar consonants were preprocessed by rotating the data from the tongue blade coil so that horizontal displacement in the rotated data would roughly correspond to movement parallel to the contour of the hard palate in the vicinity of the constriction. In other words, for each speaker an angle was determined that would orient this portion of the hard palate horizontally. Figure 2 shows speaker M's movement pattern for /odo/ (egressive) in its original orientation, before rotation.

Secondly, since we could not be sure in advance what parameter would best capture potential differences in movement paths, we made ensemble averages (usually of 10 repetitions) of tongue movement for each speaker and experimental condition. The line-up point was the time of the vertical maximum of tongue position during the consonant.

Figure 3 shows examples of ensemble-averaged trajectories for two egressive/ingressive pairs: /ogo/ at loud intensity for speaker $\mathrm{F}$ (rear tongue transducer) and /odo/ at normal intensity for speaker $\mathrm{M}$ (front tongue transducer, after rotation, see fig. 2).

Inspection of the ensemble plots suggested that the following measurement criterion would sensitively reflect the differences between ingressive/egressive movement patterns of the kind apparent in figure 3 and could be consistently applied to all speakers and conditions: the difference in horizontal position ('X DIF') of the tongue was determined at the points moving into and away from consonantal closure where the tongue was $2 \mathrm{~mm}$ below its maximum vertical position (indicated in the ensemble average plots by a horizontal dashed line). Typically this resulted in the selection of points on the movement trajectory occurring on the order of $30 \mathrm{~ms}$ earlier and later than the acoustically determined times of consonantal closure and release, respectively. Since intraoral air pressure does not decline instantaneously to zero at the release of stop consonants, the time interval defined by this criterion may reflect quite well the period over which increased air pressure is impinging on the tongue. In the absence of intraoral pressure measurements we could not, of course, test this idea directly; however, it is interesting to note that both the ingressive examples in figure 3 show strongly perturbed trajectories (with respect to the egressive case) in the vicinity of the consonantal constriction but then show signs (particularly for /odo/) of reverting to the trajectory followed in the egressive case once the tongue has lowered to about 2 or $3 \mathrm{~mm}$ below its maximum height. Practical considerations also indicated that the chosen criterion would be more robust than one based on the selection of time instants of closure and release from the acoustic waveform. Figure 3 shows that tongue movement is predominantly vertical when the trajectory crosses the dashed line indicating the 2-mm criterion. Thus mesurements of horizontal displacement will be comparatively unaffected by slight uncertainties in the time instant selected for analysis. This is much less true at acoustically defined closure and release. Firstly, these points tend to be located in more horizontally oriented portions of the trajectory (especially the release phase of velar stops); secondly, localization of an acoustically defined instant of closure formation would indeed have involved some uncertainty for the ingressive items. 


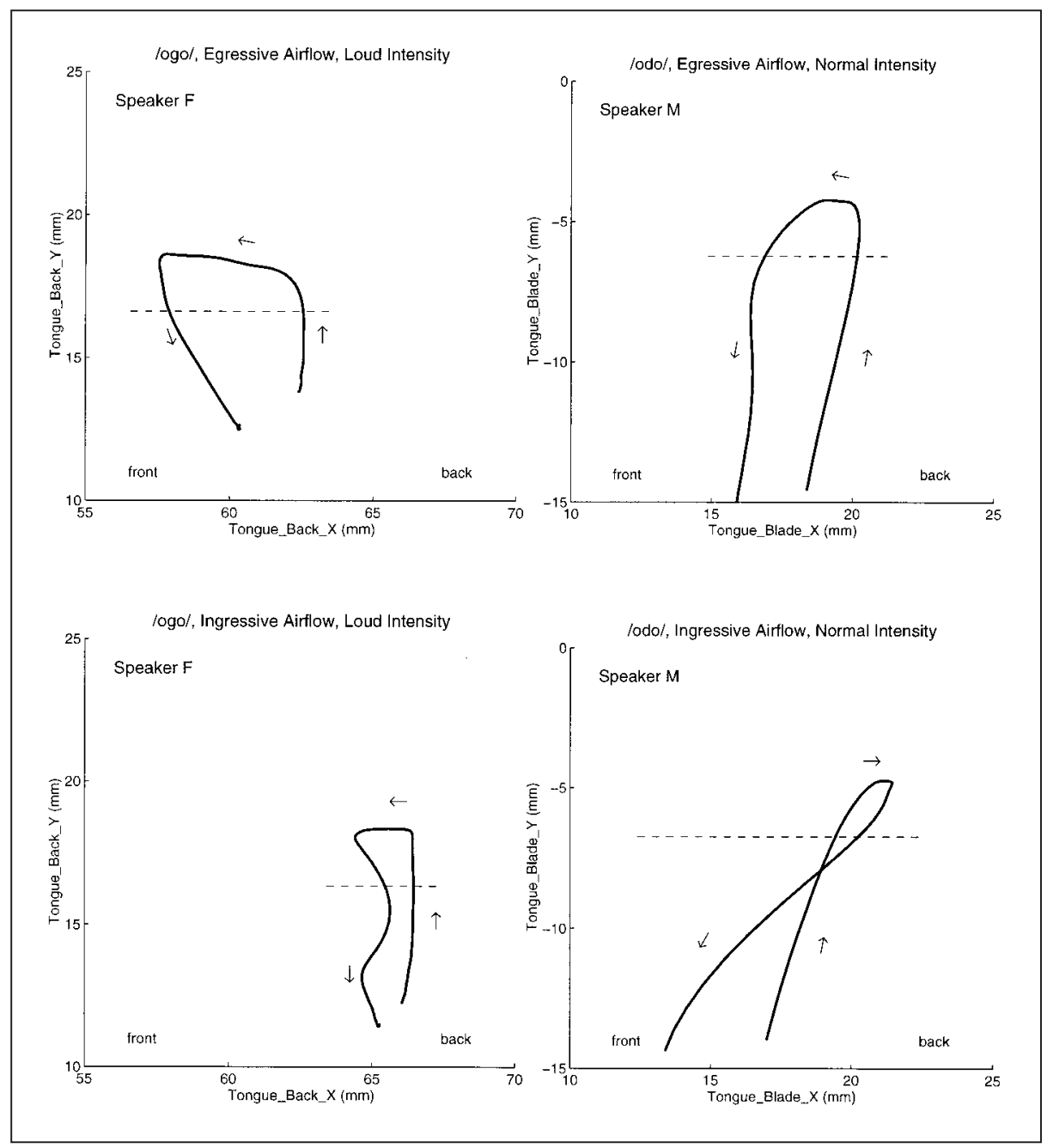

Fig. 3. Examples of ensemble-averaged tongue movement in egressive speech (top panels) and ingressive speech (bottom panels). Left panels: velar utterances, speaker F; right panels: alveolar utterances, speaker M. Movement analysis was based on points at which the dashed line intersects the movement trajectory ( $2 \mathrm{~mm}$ below maximum height).

\section{Respitrace Measurements}

The average rate of change of the thoracic and abdominal signals was calculated over the central portion of each utterance: roughly from the midpoint of $\mathrm{V}_{1}$ to the midpoint of $\mathrm{V}_{2}$. These two simple parameters seemed adequate to capture the relevant respiratory activity since preliminary inspection of the raw data had shown that the rate of change of the respiratory signals nearly always remained fairly constant over this central portion of the utterances. Figure 4 shows typical traces for egressive and ingressive utterances of speaker $\mathrm{M}$. 


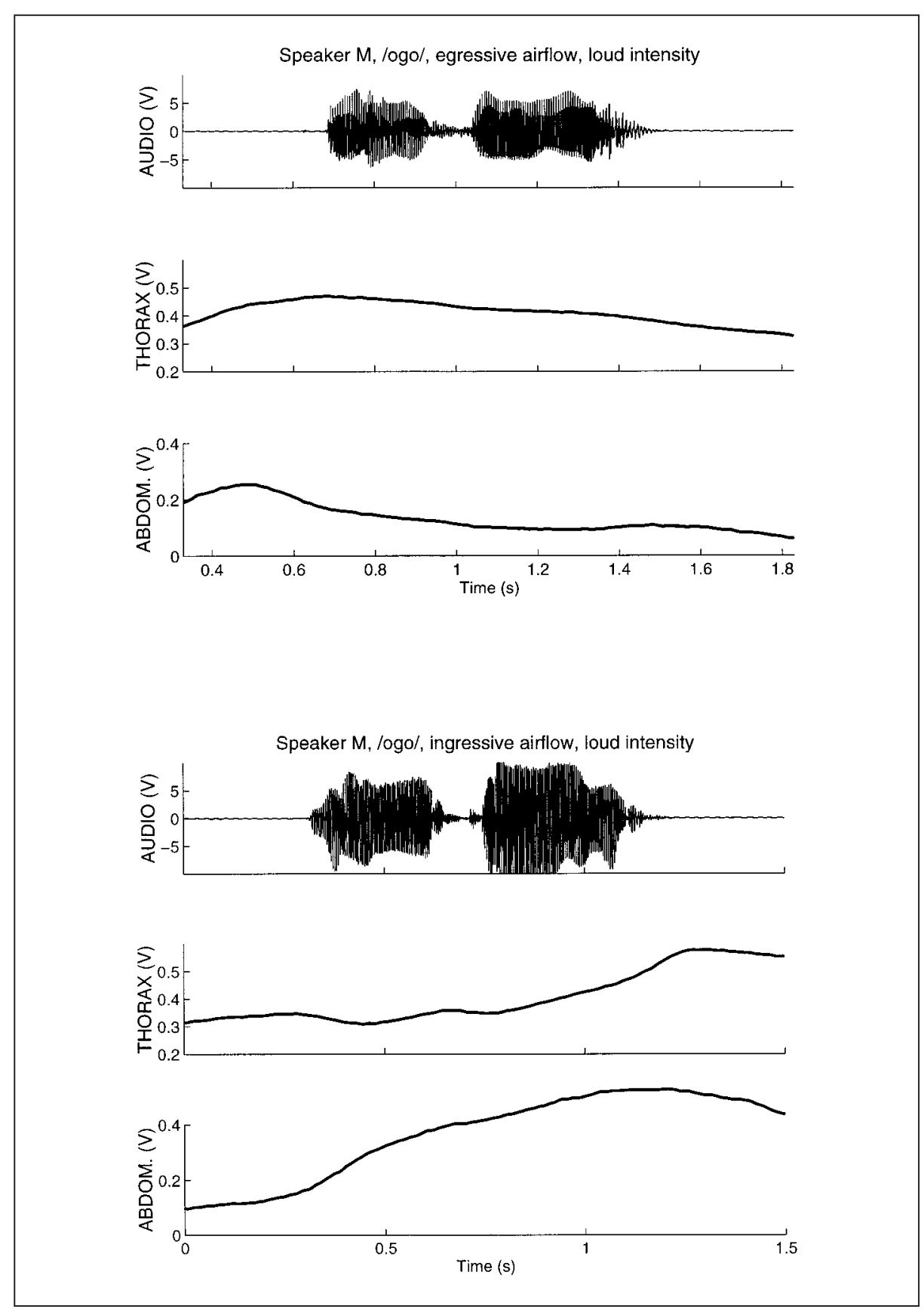

Fig. 4. Example traces of respiratory activity for egressive (top) and ingressive (bottom) /ogo/ of speaker M. 

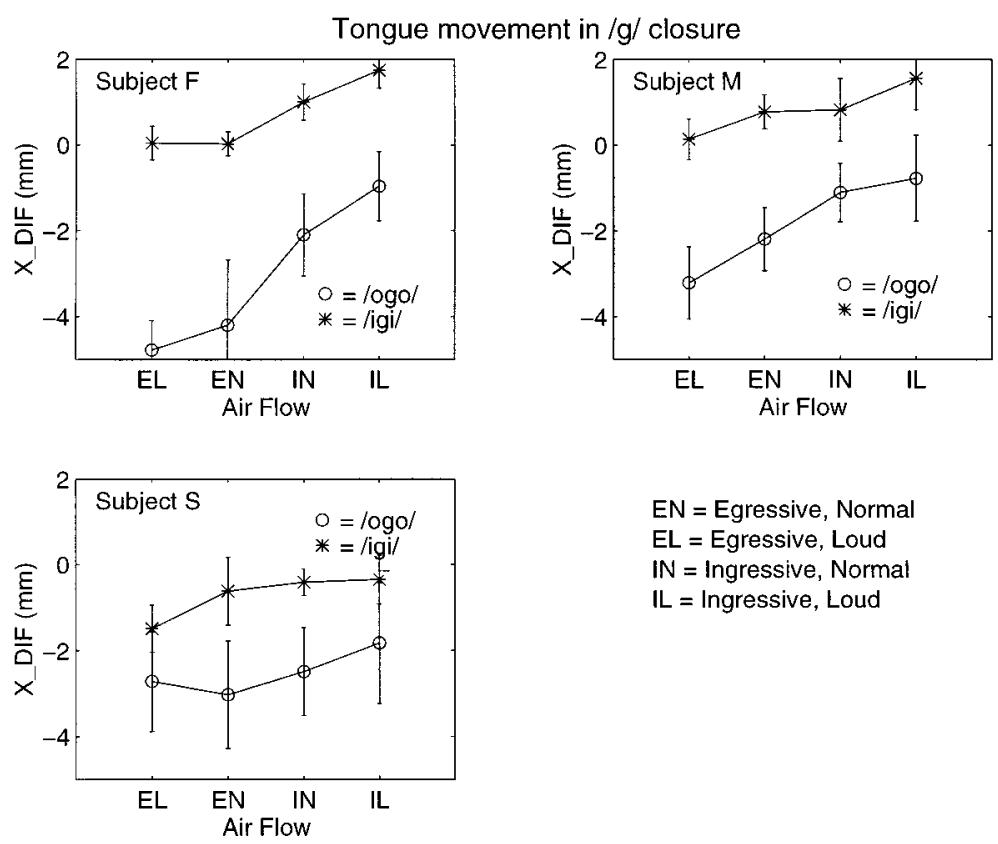

EN = Egressive, Normal

$\mathrm{EL}=$ Egressive , Loud

IN = Ingressive, Normal

$\mathrm{IL}=$ Ingressive, Loud

Fig. 5. Means and standard deviations $(n=10)$ of horizontal tongue movement over the closure phase for the target consonant /g/. Arrangement of airflow conditions on the abscissa (from left to right): egressive loud (EL), egressive normal (EN), ingressive normal (IN), ingressive loud (IL).

\section{Results}

\section{Lingual Movement}

The two ingressive/egressive pairs used above (fig.3) to illustrate the measurement criteria for tongue movement paths give representative examples of cases where variation in airflow conditions was indeed accompanied by appreciable differences in tongue movement. The /ogo/ example shows in the ingressive condition a substantial reduction in forward movement of the tongue during elevation for velar closure and even a slight retraction of the tongue as it starts to lower for the following vowel. The /odo/ example actually shows a reversal of the direction of movement in the vicinity of the consonantal constriction, from forwards in the egressive case to backwards in the ingressive one. As just noted above regarding the choice of measurement criterion, it is interesting that once the constriction has widened to about $3 \mathrm{~mm}$, the tongue shows evidence in the ingressive examples of reverting to the path it followed in the egressive counterpart, rather than following the straightest line to the following vowel.

The relationship between tongue movement and airflow condition is summarized for the material involving the target consonants $/ \mathrm{g} /$ and /d/ in figures 5 and 6 , respectively, and for the control condition involving the nasal $/ \mathrm{n} /$ in figure 7 . The means and standard deviations from the individual trajectories are plotted for the horizontal 

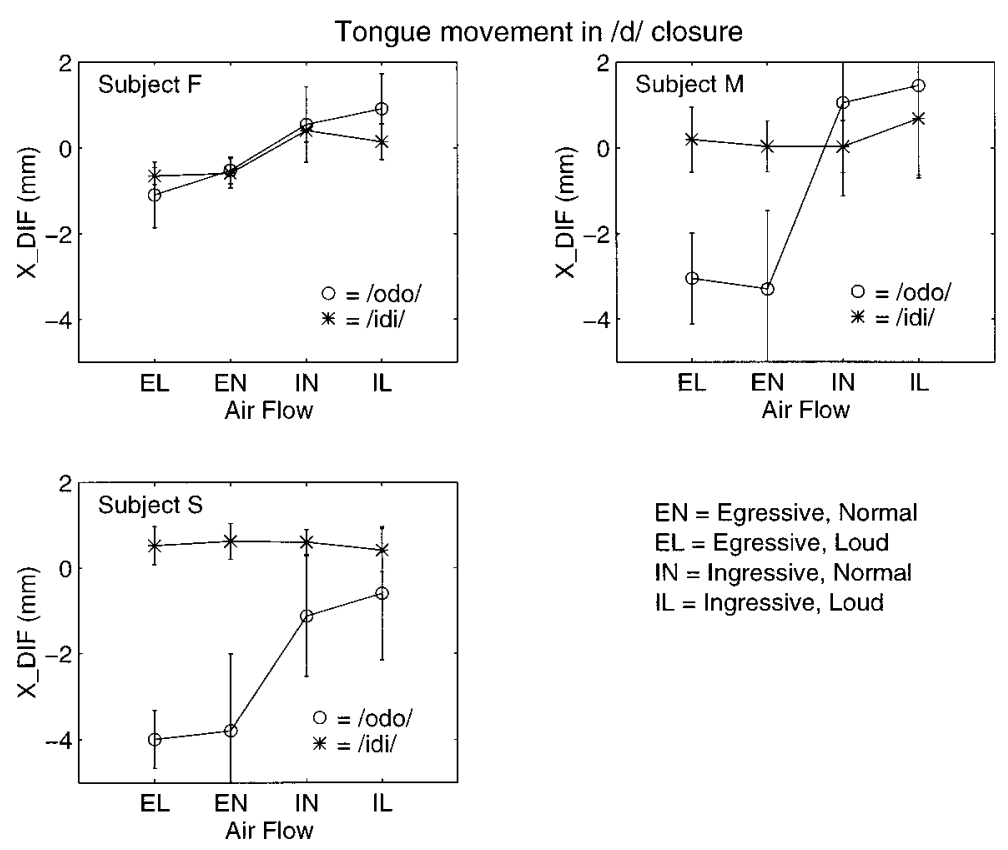

$\mathrm{EN}=$ Egressive, Normal

$\mathrm{EL}=$ Egressive, Loud

IN = Ingressive, Normal

$\mathrm{IL}=$ Ingressive, Loud

Fig. 6. Horizontal tongue movement over the closure phase for the target consonant $/ d /$. See figure 5 for details.

movement of the tongue over the consonantal closure phase (calculated according to the criterion outlined above). Negative values of this parameter indicate forward movement of the tongue. The data have been arranged on the abscissa in terms of an assumed scale of airflow, i.e. from 'loud egressive' on the left through 'normal egressive' and 'normal ingressive' to 'loud ingressive' on the right (the actual realization of the ingressive/ egressive and loud/normal contrasts in respiratory terms will be looked at in the respiratory activity section below).

Statistical testing proceeded in three stages. At the first stage, the aim was simply to confirm the overall impression that differences in airflow condition can be accompanied by statistically significant differences in the amount of horizontal tongue movement. To this end, the two independent variables 'airflow direction' (egressive vs. ingressive) and 'loudness' (normal vs. loud) were combined into a single fourlevel factor that we will refer to as 'flow' (corresponding to the four labeled positions on the abscissa in figures 5-7 just outlined above). We then carried out 6 separate twoway ANOVAs with 'flow' as the first factor and 'vowel' (/o/ vs. /i/) as the second factor. Each of these analyses corresponds to one of the panels in figures 5 and 6 (i.e. one analysis for each of 3 subjects and 2 consonants). There was a highly significant effect of flow ( $p<0.01)$ in each of these 6 cases. However, there was also a significant interaction between flow and vowel in 5 cases (with $p<0.01$ for 3 of these); this was related to the overall more restricted effect of flow in the vowel context $/ \mathrm{i} /$. 


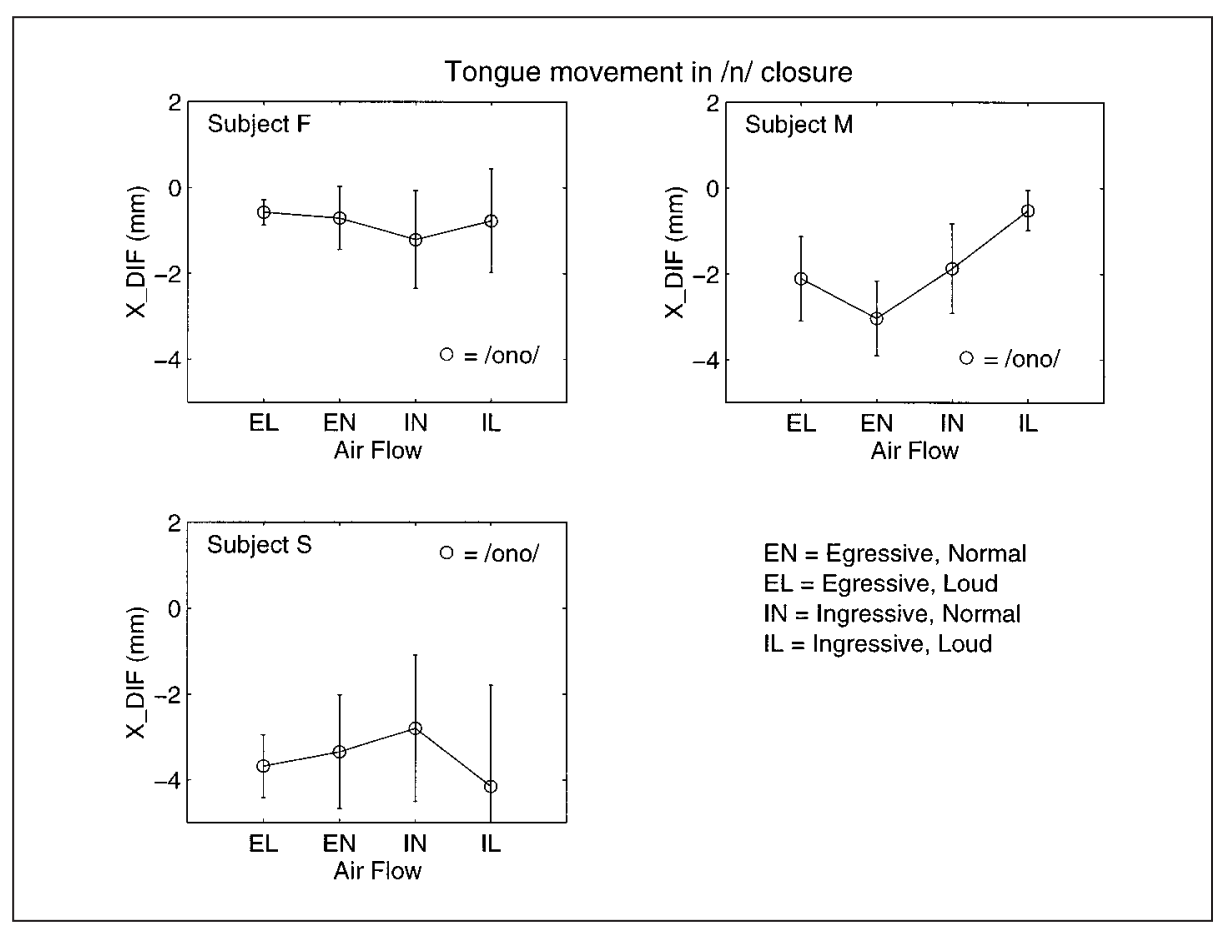

Fig. 7. Horizontal tongue movement over closure phase for target consonant $/ \mathrm{n} /$. See figure 5 for details.

The aim at the second stage of statistical testing was to determine more precisely the source and direction of the significant results related to airflow conditions. Twoway ANOVAs were carried out using the original factors of 'airflow direction' and 'loudness'.

In view of the interaction between flow and vowel found at the first stage, a separate analysis was performed for each VC (and subject) combination, i.e. a total of 12 analyses. The main effect of airflow direction was significant at $\mathrm{p}<0.01$ in 9 out of 12 cases (the exceptions being /idi/ for speakers $\mathrm{M}$ and $\mathrm{S}$, and /ogo/ for speaker $\mathrm{S}$ ). The egressive-ingressive contrast can thus be regarded as producing robust effects, with less forward tongue movement in the ingressive case. According to the above discussion in the introduction, the other main prediction for the ANOVA results is a significant interaction between airflow direction and loudness. This prediction is based on the assumption that aerodynamic conditions are relevant for tongue movement: manipulation of loudness should affect the dependent variable in opposite ways for the two airflow directions. Loud voice (compared to normal) should result in increased forward movement during the closure with egressive airflow and reduced forward movement or increased backward movement with ingressive airflow.

This expectation was not completely fulfilled. For the velar consonants (fig. 5) the interaction between airflow direction and loudness was indeed significant at at least the $5 \%$ level in 5 out of 6 cases (reaching $p<0.01$ in 2 of these 5 ), but for the alve- 
olar consonants (fig. 6) no significant interaction was obtained. Part of the reason for this is probably that the effect of the loudness manipulation was overall relatively weak compared to the airflow direction manipulation (cf. the respiratory activity section below). Thus it is not universally the case that loud voice results in increased forward movement when coupled with egressive airflow and in reduced forward movement with ingressive airflow. Indeed, a posteriori comparisons showed only one case of a significant difference between a normal vs. loud pair. Despite this, the loudness manipulation remains crucial to the experimental design since the effect of airflow direction still emerges more clearly in the loud condition. In other words, the majority of cases in figures 5 and 6 show a more obvious difference in horizontal tongue movement for loud egressive vs. loud ingressive than for normal egressive vs. normal ingressive conditions.

The first two stages of statistical testing confirmed the presence of statistically significant differences in horizontal tongue movement related to airflow conditions. For the third and final stage we now consider the control sequences with a nasal target consonant (/ono/; fig. 7). The results for these sequences are important in order to help rule out the possibility that the results for the utterances with target consonants $/ \mathrm{g} / \mathrm{and}$ /d/ were not simply due to some unknown factor that happened to covary with the airflow conditions.

The simplest pattern of results that could be expected for the /ono/ utterances is that the aerodynamic manipulations simply lead to no significant effects. This in fact occurred for speakers $\mathrm{F}$ and $\mathrm{S}$. For speaker $\mathrm{M}$ there was a significant effect of airflow direction at $p<0.05$. (There was also a significant effect of loudness, but no loudness $\times$ airflow direction interaction.) The occurrence of a significant airflow effect in the nasal sequence does not immediately invalidate the idea that the effects in the nonnasal sequences reflect aerodynamic influences, since the different aerodynamic conditions used in the experiment may still covary with other articulatory variables that are equally present in nasal and nonnasal sequences. However, we do need to be able to show, at least for this subject, that the effects of the aerodynamic manipulations are greater in the nonnasal than in the nasal case. This can be approached most directly by comparing the /ono/ utterances shown in figure 7 with the corresponding nonnasal sequences in figure 6 , i.e. /odo/. This comparison reveals for speaker M (as well as for the other two speakers) a pattern that is perfectly consistent with the expectation that sequences with a nasal consonant should be less affected by airstream conditions than oral consonants: a slightly more forward movement is observed for egressive /odo/ than for egressive /ono/, and clearly less forward movement for ingressive /odo/ than ingressive /ono/. The simplest statistical test for the significance of this pattern would be to look in a two-way ANOVA for an interaction between the factor 'flow' (the four-level factor used above for the first stage of the statistical tests) and the factor 'nasality' (a two-level factor corresponding to target consonant $/ \mathrm{n} /$ and $/ \mathrm{d} /$, respectively). This indeed occurs.

To summarize this section, it can be said that differences in aerodynamic conditions in the vocal tract are consistently accompanied by differences in horizontal tongue movement during consonantal closure. However, it is worth noting in conclusion that in our /ogo/ sequences, which correspond to the type of context in which elliptical tongue patterns have been most consistently observed in the past, that even the loud ingresive condition does not result in a reversal of tongue movement direction in the closure phase. The amount of movement is substantially reduced, but the direction remains forwards. 


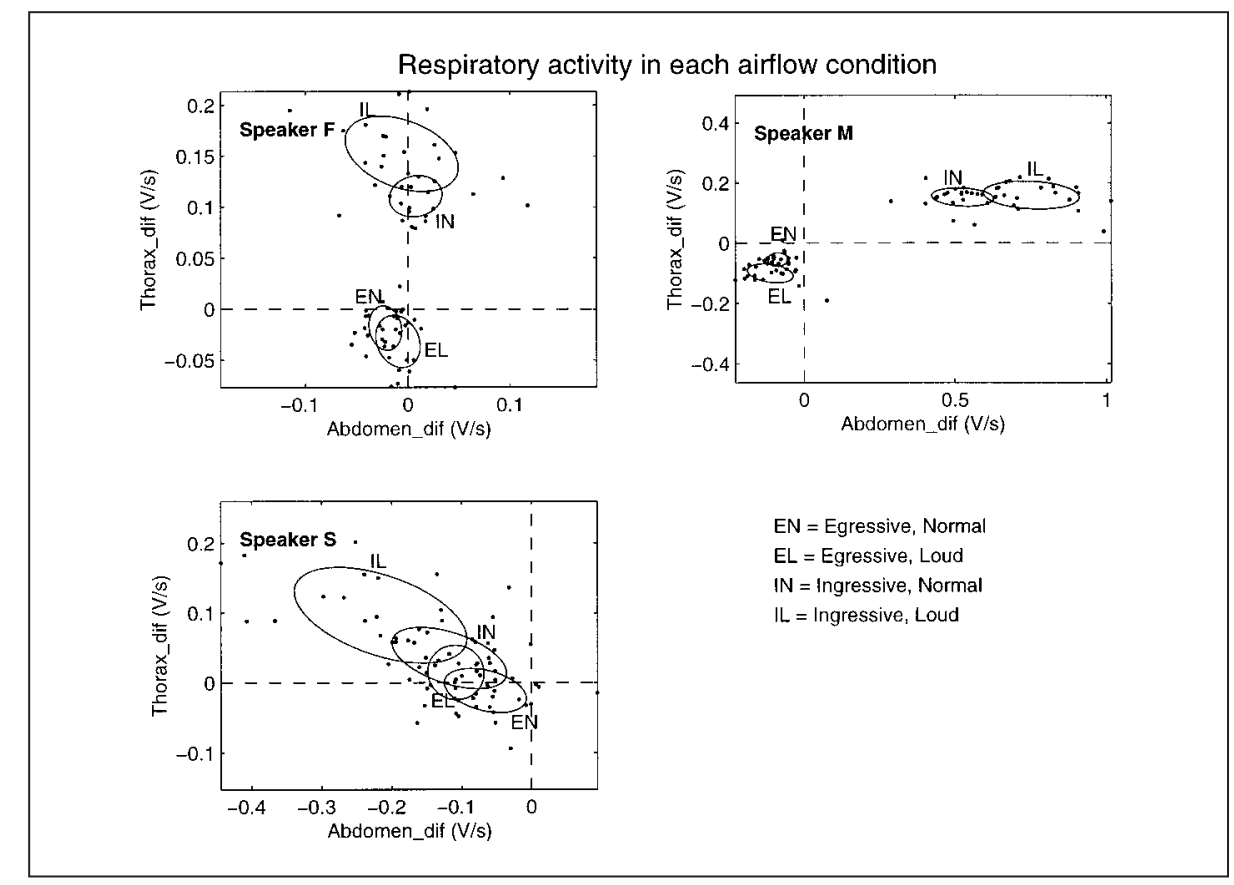

Fig. 8. Rate of change of abdominal and thoracic configuration in each airflow condition; 1 -sigma ellipses, velar target consonant, $\mathrm{n}=80$. Separate panels for each subject. For speakers M and $\mathrm{S}$, the abdominal data have been weighted to reflect volume contributions equivalent to the thoracic data. For speaker $\mathrm{F}$ the data are unweighted.

\section{Respiratory Activity}

The main aim of the present section is simply to confirm that the speakers did indeed engage in respiratory activity consistent with the requirements of the experimental conditions - a fact we implicitly assumed in the last section.

The respiratory pattern employed by each subject for each of the four airflow conditions is shown in figure 8 . The rate of change of the abdominal configuration is plotted versus the rate of change of the thoracic configuration (arbitrary units of volts per second; positive values indicate inspiratory activity, i.e. increasing respiratory volume).

Two points emerge clearly: firstly, respiratory activity for the ingressive tokens is rather vigorous compared to the egressive tokens (in fact the mesurements for the normal-intensity egressive tokens were hardly above the noise level for the particular sensitivity level to which the equipment was set in this experiment). For speakers $\mathrm{F}$ and $\mathrm{M}$, the differences between loud and normal speech are quite small compared to the differences between ingressive and egressive speech. Nonetheless, the loud versus normal contrast does show a consistent trend in the expected direction; for both airstream directions loud utterances are located further from the origin, indicating more vigorous respiratory activity (the actual differences in loudness between the loud and normal condition were of the order of $6 \mathrm{~dB}$ for all speakers and both airstream directions). 


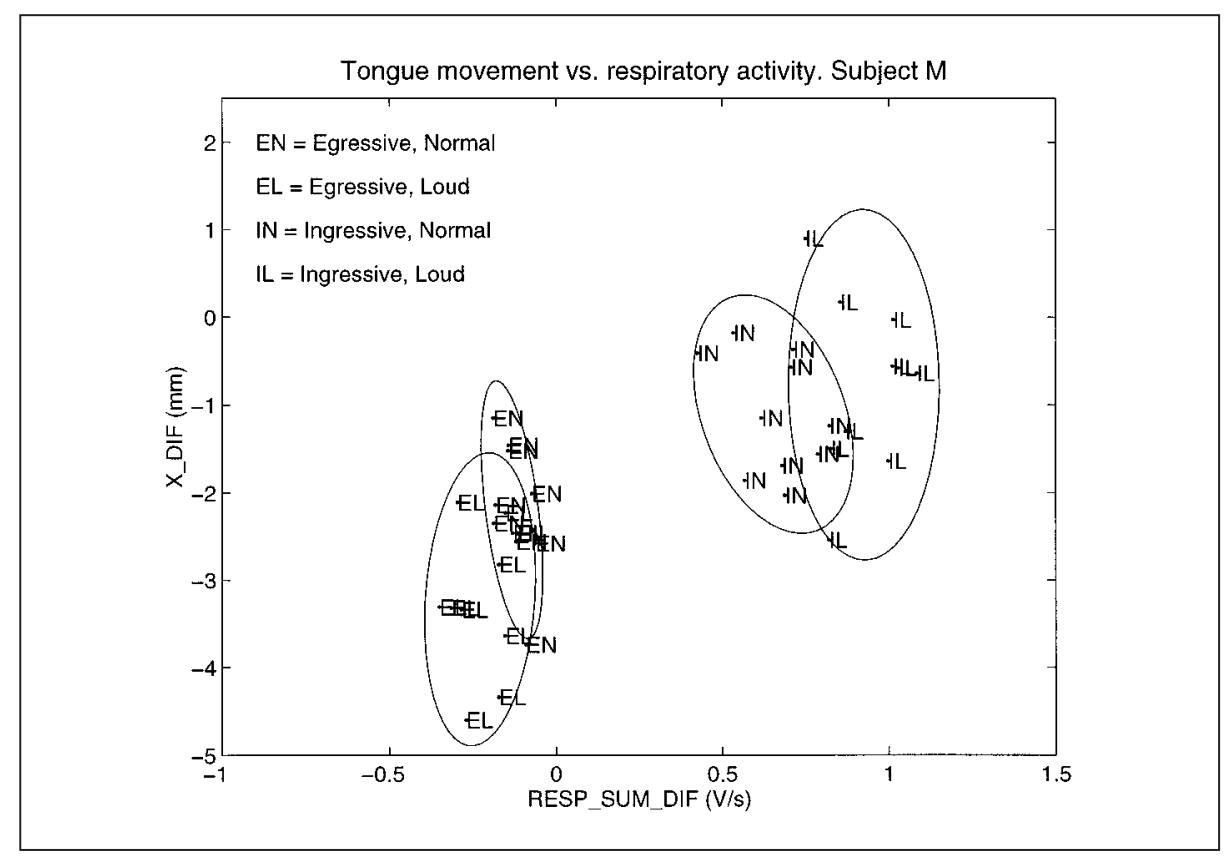

Fig. 9. Tongue movement plotted as a function of estimated overall respiratory activity ('Resp Sum Dif'). Separate 2-sigma ellipses for each respiratory condition $(\mathrm{n}=10)$. /ogo/ utterances, speaker M.

Secondly, the subjects differ radically in the respiratory adjustment used when moving from the egressive to the ingressive airstream. Speaker $M$ shows the most straightforward patterns, since for the ingressive tokens both abdominal and thoracic components change to an inspiratory direction of movement. The change from egressive to ingressive is more marked in the abdominal component, however, and he also makes more use of the abdominal component to distinguish intensity levels on the ingressive airstream. For speaker $\mathrm{F}$ the data must be interpreted with caution since the relative weight of the abdominal and thoracic contributions is not known; however, it is clear that the distributions of the data for the four experimental conditions overlap much more with respect to the abdominal component than with respect to the thoracic component. Accordingly, there appears to be no consistent change in abdominal activity going from egressive to ingressive, but there is a clear change in the inspiratory direction for the thoracic component. The pattern of speaker $\mathrm{S}$ is curious, since he shows a marked increase in inspiratory thoracic activity for the loud ingressive tokens, but at the same time a marked increase in abdominal activity in the expiratory direction. It is interesting to note that this ambiguous respiratory pattern occurs in the speaker who failed to show consistent airstream effects in the velar consonants (his relevant utterances did sound clearly ingressive however).

\section{Relationship between Lingual and Respiratory Activity}

Given the general pattern of results for horizontal movement of the tongue, i.e. a decline in forward movement moving from egressive to ingressive, the question arises 
as to how close the relationship between tongue movement and respiratory activity will be, not just in terms of averages per condition, but also on a token-to-token basis. Figure 9 shows a scatterplot of horizontal tongue movement for the /ogo/ sequences of speaker $\mathrm{M}$ as a function of overall respiratory activity (weighted sum of abdominal and thoracic components based on the isovolume maneuvers).

Over the complete material the correlation between the two variables would amount to 0.71 (Pearson's r). However, this is of dubious validity given the clearly bimodal distribution of the data. Within each condition there is no evidence of a correlation at all, despite quite wide ranges of variation in the amount of horizontal tongue movement. Consequently, there is little in the data to suggest a direct relationship between aerodynamic activity and horizontal tongue movement on an utteranceby-utterance basis. This was typical of the results for speakers $\mathrm{M}$ and $\mathrm{F}^{1}{ }^{1}$ Speaker $\mathrm{S}$ was not analyzed in view of his ambiguous respiratory patterns.

The absence of a clear relationship between respiratory drive and horizontal tongue movement at the level of individual tokens could simply reflect the fact that measurements of respiratory kinematics are a rather indirect way of getting at air pressure relationships in the vocal tract. On the other hand, it could also mean that the relationship between airstream mechanisms and tongue movement should not be interpreted as a simple mechanical effect of air pressure impinging on the tongue.

\section{Discussion}

Changes in airstream conditions were often accompanied by clear changes in tongue movement pattern. The changes might have been even more pronounced if we had used voiceless aspirated stops rather than voiced stops, since Mooshammer et al. [1995] found more forward movement for $/ \mathrm{k} /$ than for $/ \mathrm{g} /$ (we preferred to use /g/ in the present experiment, since ingressive sequences with voiced-voiceless transitions appeared difficult to produce consistently).

Air pressure conditions in the vocal tract may thus be partly responsible for elliptical patterns in speech movement (the fact that the effects were rather restricted in the context of /i/ may be a simple biomechanical consequence of the whole of the front part of the tongue being so highly constrained for the production of this vowel that there is little leeway for external forces to perturb tongue tip or tongue dorsum movement during the consonant).

On the other hand, air pressure does not appear to be solely responsible for these effects. In the velar nasals investigated by Mooshammer et al. [1995], the elliptical pattern was weakened, but not eliminated, compared with the velar stops. Similarly, many of the alveolar nasals in the present experiment had an appreciable amount of forward movement in the closure phase (i.e. values of X DIF generally negative; e.g. fig.7). Moreover, reversing the airstream from egressive to ingressive in the velar stops did not eliminate forward movement during closure but only reduced it. Of particular interest are cases such as /odo/ in figure 3 where the ingressive condition may

${ }^{1}$ As no isovolume data were available for speaker $\mathrm{F}$ (see section above), we examined horizontal tongue movement both as a function of thoracic activity alone (this being the respiratory dimension he made most systematic use of) and as a function of an unweighted combination of the thoracic and abdominal components; there was no appreciable difference in the results. 
strongly perturb the movement path in the immediate vicinity of the consonant, but where the overall V-to- $\mathrm{V}$ movement then reverts to the elliptical path found in the egressive case.

Taken together, these observations suggest that the elliptical movement patterns found in speech must be put down to at least two factors: firstly, aerodynamic factors operating in the vicinity of a consonantal constriction; secondly, asymmetries in the muscle forces responsible for $\mathrm{V}$-to- $\mathrm{C}$ and $\mathrm{C}$-to- $\mathrm{V}$ movements.

We assume that in sequences such as /ogo/ these factors operate in essentially the same direction (in the normal egressive case). Thus the familiar elliptical pattern becomes firmly established.

It is also conceivable that additional factors beyond these two may be at work in specific languages. Thus while the explanation of forward tongue movement as a mechanism to sustain voicing [Ohala, 1983] does not appear tenable for German and English we would not want to rule out the possibility that it can be used to this end in languages requiring more pronounced voicing in stops (e.g. discussion in Mooshammer et al. [1995]).

Finally we believe that it is necessary to consider more precisely in what way the aerodynamic conditions actually influence tongue movement. We have already indicated in the previous section ('relationship between lingual and respiratory activity') that it may not be correct to view overpressure in the vocal tract as directly and mechanically pushing the tongue. Evidence from other classes of movements (e.g. arm movements, grip force) suggests that the neuromotor system plans movements with a detailed knowledge of the force environment in which the movements will be produced. As a result, the motor planning system takes into account and in some cases takes advantage of the reactive forces that occur during movement production. For example, when an object such as a glass is held between the thumb and forefinger during an arm raising movement, the load force on the object increases and the grip force must also increase or the object will be dropped. Flanagan and Wing [1997] have shown that the increase in load force is anticipated and grip force modulation is synchronized with the changes in load. This result suggests that the nervous system has an internal model of the motor apparatus and the forces it will encounter. This internal model is used in motor planning to produce the desired paths of movement in spite of complex dynamic conditions [Kawato and Gomi, 1992; Miall et al., 1993; Shadmeh and Mussa-Ivaldi, 1994; Flanagan and Wing, 1997]. Similar anticipatory adjustments to external and internal forces have been demonstrated in arm movements, locomotion and postural adjustments.

In speech there has been little attention paid to the forces in speech production. The results in the present experiment suggest that this may be a fruitful line of study. In both ingressive and egressive speech the motor planning system may be anticipating the aerodynamic forces and planning movement trajectories to take advantage of the direction and magnitude of the force vector.

\section{Acknowledgement}

Thanks are given to John Ohala and one anonymous reviewer for helpful comments. 


\section{References}

Alfonso, P.; Baer, T.: Dynamics of vowel articulation. Lang. Speech 25: 151-173 (1982).

Bless, D.; Hunker, C.; Weismer, G.: Comparison of non-invasive methods to obtain chest-wall displacement and aerodynamic measures during speech; in Lawrence, Transcripts of the tenth symposium: care of the professional voice, pp. 43-51 (The Voice Foundation, New York 1982).

Cohn, M.; Watson, H.; Weisshaut, R.; Stott, F.; Sackner, M.: A transducer for non-invasive monitoring of respiration; in Stott, Raftery, Sleight, Goulding, ISAM 1977 1977: proceedings of the Second International Symposium on Ambulatory Monitoring, pp. 119-128 (Academic Press, London 1978).

Flanagan, J.R.; Wing, A.: The role of internal models in motion planning and control: evidence from grip force adjustments during movements of hand-held loads. J Neurosci. 17: 1519-1528 (1997).

Hixon, T.; Goldman, M.; Mead, J.: Kinematics of the chest wall during speech production: volume displacements of the rib cage, abdomen and lung. J. Speech Hear. Res. 16: 78-115 (1973).

Hoole, P.: Methodological considerations in the use of electromagnetic articulography in phonetic research. Forschungsber. Inst. Phonetik sprachl. Kommun. München 31: 43-64 (1993).

Hoole, P.: Issues in the acquisition, processing, reduction and parameterization of articulographic data. Forschungsber. Inst. Phonetik sprachl. Kommun. München 34: 158-173 (1996).

Houde, R.A.: A study of tongue body motion during selected speech sounds. Speech Communication Research Laboratory, Santa Barbara, Monograph No. 2 (1968).

Kawato, M.; Gomi, H.: A computational model of four regions of the cerebellum based on feedback-error learning. Biol. Cybernet. 68: 95-103 (1992).

Kent, R.; Moll, K.: Cinefluorographic analyses of selected lingual consonants. J. Speech Hear. Res. 15: 453-473 (1972).

Miall, R.C.; Weir, D.J.; Wolpert, D.M.; Stein, J.F.: Is the cerebellum a Smith predictor? J. Motor Behav. 25: 203-216 (1993).

Mooshammer, C.; Hoole, P.; Kühnert, B.: On loops. J. Phonetics 23: 3-21 (1995).

Munhall, K.; Ostry, D.; Flanagan, J.: Coordinate spaces in speech planning. J Phonetics 19: 293-307 (1991).

Ohala, J.J.: The origin of sound patterns in vocal tract constraints; in MacNeilage, The production of speech, pp. 189-216 (Springer, New York 1983).

Perkell, J.S.: Physiology of speech production: results and implications of a quantitative cineradiographic study (MIT Press, Cambridge 1969).

Perkell, J.; Cohen, M.; Svirsky, M.; Matthies, M.; Garabieta, I.; Jackson, M.: Electromagnetic midsagittal articulometer (EMMA) systems for transducing speech articulatory movements. J. acoust. Soc. Am. 92: 3078-3096 (1992).

Sanguineti, V.; Laboissière, R.; Ostry, D.J.: A dynamic biomechanical model for neural control of speech production. J. acoust. Soc. Am. 103: 1615-1627 (1998).

Shadmeh, R.; Mussa-Ivaldi, A.: Adaptive representation of dynamics during learning of a motor task. J. Neurosci. 14: 3208-3224 (1994).

Svirsky, M.; Stevens, K.; Matthies, M.; Manzella, J.; Perkell, J.; Wilhelms-Tricarico, R.: Tongue surface displacement during bilabial stops. J. acoust. Soc. Am. 102: 562-571 (1997). 